\title{
Postoperative Short-term Outcomes Between Sublobar Resection and Lobectomy in Patients with Lung Adenocarcinoma
}

This article was published in the following Dove Press journal: Cancer Management and Research

\author{
Jiaqi Zhang $\mathbb{C}^{*}$ \\ Wenliang Bai* \\ Chao Guo ID \\ Lei Liu \\ Guige Wang (iD \\ Cheng Huang \\ Yeye Chen \\ Ye Zhang \\ Shanqing $\mathrm{Li}$
}

Department of Thoracic Surgery, Peking Union Medical College Hospital, Chinese Academy of Medical Sciences and Peking Union Medical College, Beijing 100730,

People's Republic of China

*These authors contributed equally to this work
Correspondence: Shanqing Li

$\mathrm{Tel}+86$ I3/21971623

Fax +861069152630

Email13121971623@163.com
Background: To investigate postoperative temporary consequences of the enrolled patients with lung adenocarcinoma.

Patients and Methods: We analyzed the clinical data of patients with lung adenocarcinoma admitted by the same surgical team of Peking Union Medical College Hospital (PUMCH) from July 2019 to December 2019. Statistical methods including propensity score matching (PSM) analysis was used to analyze the differences among them.

Results: A total of 108 patients were enrolled, including 50 patients with sublobar resection and 58 patients with lobectomy. Before PSM, there were statistically significant differences in age $(p=0.015)$, hospitalization costs $(p=0.042)$, lymphadenectomy $(p=0.000)$, pathological staging $(p=0.000)$, number of lymph nodes removed $(p=0.000)$, number of positive lymph nodes $(p=0.034)$, chest drainage duration $(p=0.000)$, total chest drainage $(p=0.000)$, length of postoperative hospital stays $(p=0.000)$, postoperative $\mathrm{D}$-dimer level $(p=0.030)$ and perioperative lymphocyte margin (LM) $(p=0.003)$ between sublobar resection and lobectomy. After PSM, there were statistical differences in number of lymph nodes removed $(p=0.000)$, chest drainage duration $(p=0.031)$ and total chest drainage $(p=0.002)$ between sublobar resection and lobectomy. Whether with PSM analysis or not, there were no significant differences in other blood test results, such as inflammation indicators, postoperative neutrophil-lymphocyte ratio (NLR), albumin level, perioperative activity of daily living (ADL) scale scoring margin, complications, postoperative admission to intensive care unit (ICU) and readmission within 30 days. NLR was associated with total chest drainage $(p=0.000)$, length of postoperative hospital stays $(p=0.000)$, postoperative D-dimer level $(p=0.050)$ and ADL scale scoring margin $(p=0.003)$ between sublobar resection and lobectomy.

Conclusion: Sublobar resection, including wedge resection and segmentectomy, was as safe and feasible as lobectomy in our study, and they shared similar short-term outcomes. Postoperative NLR could be used to detect the clinical outcomes of patients. Secondary resectability of pulmonary function (SRPF) should be the main purpose of sublobar resection.

Keywords: lung adenocarcinoma, lobectomy, sublobar resection, short-term outcome

\section{Introduction}

According to global cancer statistics in 2018, lung cancer, accounting for $11.6 \%$ of all cancers, has been the most common cancer and the leading cause of cancerrelated death. ${ }^{1}$ In recent years, with the progress of cancer screening and decline in smoking, the population of lung cancer-related deaths has declined. Early 
diagnosis and early treatment significantly benefit the overall survival of lung cancer patients. The latest US cancer statistics reported that the five-year survival rate of lung cancer was $19 \% .^{2,3}$ The continuous optimization of surgery, radiotherapy, chemotherapy, targeted therapy, and immunotherapy has gradually improved the survival of lung cancer patients. Surgical treatment progresses rapidly. Based on considerations such as minor damage and preservation of normal lung function, and as a result of the early detection of lung cancer, sublobar resection gradually emerged. Studies have shown that sublobar resection had a good protective effect on patients' residual lung function. ${ }^{4}$ However, the surgical damage evaluation of patients with different surgical methods is inconclusive, and the impact of different lung resections on the short-term outcomes of patients is not yet clear. Considering that adenocarcinoma dominated a great part in lung cancer, the purpose of this study was to retrospectively analyze the short-term outcomes of lung adenocarcinoma patients who underwent different lung resections.

\section{Patients and Methods}

\section{Patients}

We retrospectively evaluated a consecutive series of patients who underwent lung resection for lung adenocarcinoma in the Department of Thoracic Surgery, PUMCH from July 2019 to December 2019. The Institutional Review Board of PUMCH approved the study. Written informed consent was obtained from all eligible patients.

All patients underwent systemic evaluation before surgery, including clinical staging evaluation and cardiopulmonary function evaluation. Preoperative cancer staging was assessed by positron emission tomography/computed tomography (CT), or chest and abdomen CT combined with enhanced brain magnetic resonance imaging/CT and whole-body bone scintigraphy. The eighth edition AJCC/ UICC lung cancer stage classification was used for staging.

Patients included met the following criteria: (1) surgeons were senior physicians in the same surgical team with similar oncological and surgical principles (one surgical team, consisting of a professor who is the director of thoracic surgery ( $>10$ years) and three attending physicians); (2) video-assisted thoracic surgery (VATS) was performed in all patients; (3) postoperative paraffin pathology confirmed lung adenocarcinoma or carcinoma in situ.
Exclusion criteria included the following: (1) preoperative neoadjuvant therapy; (2) preoperative systemic evaluation considered malignant pleural effusion, N3 lymph node metastasis or extrathoracic metastases; (3) mediastinal mass resection, esophageal repair and other nonpulmonary operations were performed simultaneously; (4) metastatic lung cancer; (5) simultaneous bilateral surgery.

\section{Surgical Technique}

VATS was a priority in all operations. If an unexpected situation occurred during the operation, such as major bleeding, the operation would be converted to thoracotomy. Lobectomy and sublobar resection were conducted according to the NCCN guidelines. ${ }^{5}$ Sublobar resection was conducted if the nodule was $\leq 2 \mathrm{~cm}$ and it met one of the following criteria: adenocarcinoma in situ, groundglass opacity (GGO) $>50 \%$ or doubling time $\geq 400$ days. Segmentectomy was preferred over wedge resection. In sublobar resection, including wedge resection and segmentectomy, stapler was used for dividing intersegmental plane. Systematic lymph node dissection was usually performed in lobectomy, and systematic lymph node sampling was often conducted in sublobar resection.

\section{Perioperative Management}

Preoperative preparations included smoking and alcohol cessation, respiratory function training and patients' education. Patients fasted for eight hours before surgery, and resumed oral fluid intake six hours after surgery. Blood was monitored on the first morning after operation. A bedside chest radiograph was conducted on the first day after surgery to assess lung retention and chest drainage status. Early mobilization with underground activities at least 2 3 times a day was encouraged, following by normal diet. Lower fat intake and appropriate protein and electrolyte supplementation during perioperative period were recommended. Drugs for pain relief, phlegm reduction, atomization and preventing infection were routinely given to patients after surgery. According to the patient's serum albumin level, we administrated them with human albumin to ensure a normal level. If chest drainage was less than $200 \mathrm{~mL} / \mathrm{d}$ after surgery, and there were no obvious air leak and chylothorax, the chest tube would be removed. A chest radiograph would be taken on the first day after extubation. Patients without obvious pneumothorax and pleural effusion could be considered for discharge. All patients were scored with the activity of daily living (ADL) scale by specialized nurses when they 
were admitted to and discharged from hospital. Outpatient follow-up for all patients was conducted about one month later.

\section{Data Collection}

Data were collected from all enrolled patients comprising basic information, surgical information, pathology, perioperative blood test results, chest drainage status, hospitalization schedule, complications, ADL scale score, postoperative admission to ICU, readmission within 30 days and hospitalization costs (before settlement of medical insurance). We calculated body mass index (BMI).

Blood test results include white blood cell (WBC) counting, neutrophil counting, lymphocyte counting, hemoglobin concentrations, platelet counting, albumin level and D-dimer level. We calculated the NLR, neutrophil-albumin ratio, platelet-D-dimer ratio, platelet-albumin ratio, D-dimer margin, albumin margin, neutrophil margin, lymphocyte margin (LM), platelet margin, WBC margin.

With respect to complications, we listed several common or serious ones, including acute coronary syndrome (ACS), pulmonary embolism (PE), atrial fibrillation (AF), pneumonia, air leak and chylothorax.

\section{Propensity Score Matching Analysis}

To minimize selection bias between two groups, a propensity score matching (PSM) analysis was performed. The ratio of patients in each group was 1:1, the match tolerance was therefore set at 0.02 , and the following variables were used for the PSM analysis: sex, age, BMI, and pathological staging.

\section{Statistical Analysis}

Statistical analysis was performed using SPSS 23.0 software (IBM Corp., Armonk, NY, USA). The measurement data were expressed as $\overline{\mathrm{X}} \pm$ s. One-way ANOVA test or rank sum test was used to compare the differences between measurement data. Count data were expressed as percentages, and comparisons between groups were performed using chi-squared test. A two-tailed $p<0.05$ was considered statistically significant. The correlation of measurement data was analyzed by rank correlation test.

\section{Results}

A total of 108 patients with lung adenocarcinoma were enrolled, including 31 males and 77 females, with average age of $(57.09 \pm 12.00)$ years. Among them, 50 patients had sublobar resection and 58 patients underwent lobectomy.
Patients were classified into two groups according to different lung resections, and the demographics of patients in each group are shown in Table 1. All patients had no perioperative blood transfusion.

Female patients accounted for $71.30 \%$ of enrolled patients, but there was no statistically significant difference in sex between sublobar resection and lobectomy $(p=0.316)$. There was statistical difference in terms of age $(p=0.015)$.

There were statistically significant differences in lymphadenectomy $(p=0.000)$ and pathological staging ( $p=0.001)$ between sublobar resection and lobectomy. But among pathological results, spread through air spaces (STAS) $(p=0.916)$, vascular invasion $(p=0.103)$, visceral pleural involvement $(p=0.331)$, and bronchus invasion $(p=0.073)$ showed no statistically significant differences between sublobar resection and lobectomy. There were a larger number of lymph nodes removed and number of positive lymph nodes in patients with lobectomy than that in patients with sublobar resection $(p=0.000, p=0.034$, respectively).

Between sublobar resection and lobectomy, there were statistically significant differences in chest drainage duration $(p=0.000)$, total chest drainage $(p=0.000)$, length of postoperative hospital stays $(p=0.000$ ), and hospitalization costs $(p=0.042)$. But there were no statistical differences in duration of operation $(p=0.063)$ and bleeding volume during surgery $(p=0.181)$.

Postoperative D-dimer level $(p=0.030)$ and perioperative LM $(p=0.003)$ were statistically different between sublobar resection and lobectomy. There were no statistically significant differences in NLR $(p=0.170)$ and other test results between them.

Among 108 patients, three had AF, four had air leak, one had ACS and one suffered chylothorax. Two patients were admitted to ICU after operation because of ACS and one patient was admitted to ICU because of anaphylactic shock. Three patients returned to hospital within 30 days owing to recurrent pleural effusion, influenza A virus infection and lower extremity deep vein thrombosis, respectively. After symptomatic treatment, they recovered well without serious consequences. There were no significant differences among sublobar resection and lobectomy in terms of postoperative complications, postoperative admission to ICU $(p=0.473)$, readmission within 30 days $(p=0.473)$, and ADL scale scoring margin $(p=0.743)$.

After PSM analysis, sublobar resection and lobectomy showed different lymphadenectomy $(p=0.000)$, number of 
Table I Demographics of Patients Undergoing Lung Resection

\begin{tabular}{|c|c|c|c|c|c|c|c|c|}
\hline \multirow[t]{2}{*}{ Variables } & \multicolumn{4}{|l|}{ Pre-match } & \multicolumn{4}{|l|}{ Post-match } \\
\hline & $\begin{array}{l}\text { Sublobar } \\
\text { Resection }\end{array}$ & Lobectomy & SUM & $\begin{array}{l}p- \\
\text { value }\end{array}$ & $\begin{array}{l}\text { Sublobar } \\
\text { Resection }\end{array}$ & Lobectomy & Sum & $\begin{array}{l}p \text { - } \\
\text { value }\end{array}$ \\
\hline $\mathrm{N}$ & 50 & 58 & 108 & a & 32 & 32 & 64 & a \\
\hline Sex (female) & 38 & 39 & 77 & 0.316 & 24 & 24 & 48 & 1.000 \\
\hline Age (years) & $54.09 \pm 13.06$ & $59.68 \pm 10.43$ & $57.09 \pm 12.00$ & $0.015^{b}$ & $57.52 \pm 12.33$ & $57.84 \pm \mid 1.37$ & $57.68 \pm 11.77$ & 0.916 \\
\hline BMI $\left(\mathrm{kg} / \mathrm{m}^{2}\right)$ & $24.05 \pm 2.94$ & $24.15 \pm 3.08$ & $24.11 \pm 3.00$ & 0.861 & $24.03 \pm 2.91$ & $24.24 \pm 3.09$ & $24.14 \pm 2.98$ & 0.784 \\
\hline Hospitalization costs (yuan) & $\begin{array}{l}63,383.57 \\
\pm 26,102.83\end{array}$ & $\begin{array}{l}72,375.85 \\
\pm 17,620.40\end{array}$ & $\begin{array}{l}68,212.76 \\
\pm 22,305.23\end{array}$ & $0.042^{b}$ & $\begin{array}{l}71,137.95 \\
\pm 24,924.36\end{array}$ & $\begin{array}{l}70,859.02 \\
\pm 17,019.00\end{array}$ & $\begin{array}{l}70,998.49 \\
\pm 21,171.34\end{array}$ & 0.958 \\
\hline Lymphadenectomy & & & & $0.000^{\mathrm{b}}$ & & & & $0.000^{\mathrm{b}}$ \\
\hline None & 12 & 0 & 12 & & 6 & 0 & 6 & \\
\hline Sampling & 24 & 2 & 26 & & 17 & 1 & 18 & \\
\hline Dissection & 14 & 56 & 70 & & 9 & 31 & 40 & \\
\hline Pathological Staging & & & & $0.000^{\mathrm{b}}$ & & & & 1.000 \\
\hline 0 & 19 & 3 & 22 & & 3 & 3 & 6 & \\
\hline I & 30 & 46 & 76 & & 28 & 28 & 56 & \\
\hline II & 0 & 6 & 6 & & 0 & 0 & 0 & \\
\hline III & 0 & 2 & 2 & & 0 & 0 & 0 & \\
\hline IV & 1 & I & 2 & & I & 1 & 2 & \\
\hline STAS & 1 & 1 & 2 & 0.916 & I & 0 & 1 & 0.313 \\
\hline Vascular invasion & 0 & 3 & 3 & 0.103 & 0 & 1 & 1 & 0.313 \\
\hline Visceral pleural involvement & 2 & 5 & 7 & 0.331 & 2 & 2 & 4 & 1.000 \\
\hline Bronchus invasion & 3 & 10 & 13 & 0.073 & 2 & 3 & 5 & $0.64 I$ \\
\hline $\begin{array}{l}\text { Number of lymph nodes } \\
\text { removed }\end{array}$ & $10.00 \pm 8.31$ & $22.34 \pm 8.94$ & $16.63 \pm 10.61$ & $0.000^{\mathrm{b}}$ & $11.38 \pm 8.23$ & $21.50 \pm 7.74$ & $16.44 \pm 9.43$ & $0.000^{\mathrm{b}}$ \\
\hline $\begin{array}{l}\text { Number of positive lymph } \\
\text { nodes }\end{array}$ & $0.00 \pm 0.00$ & $0.2 I \pm 0.8 I$ & $0.11 \pm 0.60$ & $0.034^{\mathrm{b}}$ & $0.00 \pm 0.00$ & $0.00 \pm 0.00$ & $0.00 \pm 0.00$ & 1.000 \\
\hline Duration of operation (min) & $97.10 \pm 48.36$ & $\begin{array}{l}106.64 \\
\pm 42.11\end{array}$ & $\begin{array}{l}102.22 \\
\pm 45.15\end{array}$ & 0.063 & $\begin{array}{l}106.72 \\
\pm 50.67\end{array}$ & $\begin{array}{r}103.13 \\
\pm 25.65\end{array}$ & $\begin{array}{l}104.92 \\
\pm 39.88\end{array}$ & 0.567 \\
\hline Bleeding volume (mL) & $64.00 \pm 37.53$ & $86.72 \pm 89.80$ & $76.20 \pm 71.21$ & 0.181 & $65.00 \pm 39.43$ & $93.75 \pm 99.80$ & $79.38 \pm 76.65$ & 0.079 \\
\hline $\begin{array}{l}\text { Chest drainage duration } \\
\text { (days) }\end{array}$ & $2.12 \pm 1.47$ & $3.10 \pm 2.08$ & $2.65 \pm 1.88$ & $0.000^{b}$ & $2.50 \pm 1.44$ & $3.00 \pm 1.41$ & $2.75 \pm 1.44$ & $0.031^{b}$ \\
\hline Total chest drainage $(\mathrm{mL})$ & $\begin{array}{l}430.70 \\
\pm 488.68\end{array}$ & $\begin{array}{l}687.24 \\
\pm 405.78\end{array}$ & $\begin{array}{l}568.47 \\
\pm 462.16\end{array}$ & $0.000^{\mathrm{b}}$ & $\begin{array}{l}487.60 \\
\pm 541.59\end{array}$ & $\begin{array}{l}703.91 \\
\pm 372.69\end{array}$ & $\begin{array}{l}595.78 \\
\pm 473.87\end{array}$ & $0.002^{\mathrm{b}}$ \\
\hline $\begin{array}{l}\text { Length of postoperative } \\
\text { hospital stay (days) }\end{array}$ & $3.32 \pm 1.38$ & $4.22 \pm 2.03$ & $3.8 I \pm I .8 I$ & $0.000^{\mathrm{b}}$ & $3.63 \pm|.4|$ & $4.06 \pm 1.39$ & $3.84 \pm|.4|$ & 0.056 \\
\hline
\end{tabular}

(Continued) 
Table I (Continued).

\begin{tabular}{|c|c|c|c|c|c|c|c|c|}
\hline \multirow[t]{2}{*}{ Variables } & \multicolumn{4}{|l|}{ Pre-match } & \multicolumn{4}{|l|}{ Post-match } \\
\hline & $\begin{array}{l}\text { Sublobar } \\
\text { Resection }\end{array}$ & Lobectomy & SUM & $\begin{array}{l}p- \\
\text { value }\end{array}$ & $\begin{array}{l}\text { Sublobar } \\
\text { Resection }\end{array}$ & Lobectomy & Sum & $\begin{array}{l}p- \\
\text { value }\end{array}$ \\
\hline $\begin{array}{l}\text { Postoperative WBC counting } \\
\left(10^{9} / \mathrm{L}\right)\end{array}$ & $10.82 \pm 2.93$ & $11.34 \pm 2.63$ & $11.10 \pm 2.77$ & 0.331 & $10.92 \pm 3.22$ & $11.00 \pm 2.36$ & $10.96 \pm 2.80$ & 0.910 \\
\hline $\begin{array}{l}\text { Postoperative neutrophil } \\
\text { counting }\left(10^{9} / \mathrm{L}\right)\end{array}$ & $8.97 \pm 2.93$ & $9.51 \pm 2.59$ & $9.26 \pm 2.76$ & 0.191 & $9.20 \pm 3.22$ & $9.26 I \pm 2.40$ & $9.19 \pm 2.82$ & 0.528 \\
\hline $\begin{array}{l}\text { Postoperative lymphocyte } \\
\text { counting }\left(10^{9} / \mathrm{L}\right)\end{array}$ & $|.24 \pm 0.5|$ & $1.16 \pm 0.46$ & $1.19 \pm 0.48$ & 0.366 & $1.20 \pm 0.46$ & $1.12 \pm 0.40$ & $1.16 \pm 0.43$ & 0.438 \\
\hline $\begin{array}{l}\text { Postoperative hemoglobin } \\
\text { concentrations (g/L) }\end{array}$ & $\begin{array}{l}127.46 \\
\pm 11.34\end{array}$ & $\begin{array}{l}130.14 \\
\pm 14.55\end{array}$ & $\begin{array}{l}128.90 \\
\pm 13.17\end{array}$ & 0.294 & $\begin{array}{l}128.38 \\
\pm 12.30\end{array}$ & $\begin{array}{l}129.44 \\
\pm 13.47\end{array}$ & $\begin{array}{l}|28.9| \\
\pm|2.8|\end{array}$ & 0.743 \\
\hline $\begin{array}{l}\text { Postoperative platelet } \\
\text { counting }\left(10^{9} / \mathrm{L}\right)\end{array}$ & $\begin{array}{l}218.40 \\
\pm 45.89\end{array}$ & $\begin{array}{l}207.28 \\
\pm 39.70\end{array}$ & $\begin{array}{l}212.43 \\
\pm 42.83\end{array}$ & 0.180 & $\begin{array}{l}221.72 \\
\pm 48.69\end{array}$ & $\begin{array}{l}205.22 \\
\pm 33.81\end{array}$ & $\begin{array}{l}213.47 \\
\pm 42.41\end{array}$ & 0.120 \\
\hline $\begin{array}{l}\text { Postoperative albumin level } \\
(\mathrm{g} / \mathrm{L})\end{array}$ & $36.90 \pm 2.84$ & $36.93 \pm 2.77$ & $36.92 \pm 2.79$ & 0.526 & $36.38 \pm 3.01$ & $36.8 I \pm 3.00$ & $36.59 \pm 2.99$ & 0.807 \\
\hline $\begin{array}{l}\text { Postoperative D-dimer level } \\
\text { (mg/L FEU) }\end{array}$ & $2.24 \pm 1.85$ & $1.81 \pm 1.48$ & $2.01 \pm 1.67$ & $0.030^{\mathrm{b}}$ & $2.5 I \pm 2.16$ & $1.91 \pm 1.54$ & $2.21 \pm 1.89$ & 0.063 \\
\hline Postoperative NLR & $9.55 \pm 7.82$ & $9.97 \pm 5.74$ & $9.78 \pm 6.75$ & 0.170 & $10.07 \pm 8.65$ & $9.95 \pm 6.03$ & $10.01 \pm 7.40$ & 0.368 \\
\hline Postoperative NAR & $0.25 \pm 0.08$ & $0.26 \pm 0.07$ & $0.25 \pm 0.08$ & 0.304 & $0.25 \pm 0.09$ & $0.25 \pm 0.06$ & $0.25 \pm 0.08$ & 0.835 \\
\hline Postoperative PDR & $\begin{array}{l}164.62 \\
\pm 170.54\end{array}$ & $\begin{array}{r}167.10 \\
\pm 95.59\end{array}$ & $\begin{array}{l}165.95 \\
\pm 134.87\end{array}$ & 0.128 & $\begin{array}{l}146.26 \\
\pm 126.53\end{array}$ & $\begin{array}{l}156.33 \\
\pm 84.32\end{array}$ & $\begin{array}{l}151.29 \\
\pm 106.78\end{array}$ & 0.155 \\
\hline Postoperative PAR & $5.95 \pm 1.30$ & $5.63 \pm 1.10$ & $5.78 \pm \mid .21$ & 0.177 & $6.13 \pm 1.36$ & $5.61 \pm 1.05$ & $5.87 \pm 1.24$ & 0.097 \\
\hline Perioperative DDM & $1.85 \pm 1.87$ & $1.49 \pm 1.37$ & $1.66 \pm 1.63$ & 0.092 & $2.08 \pm 2.21$ & $1.52 \pm 1.33$ & $1.80 \pm 1.83$ & 0.118 \\
\hline Perioperative AM & $6.64 \pm 3.47$ & $6.43 \pm 2.72$ & $6.53 \pm 3.08$ & 0.926 & $6.94 \pm 3.81$ & $6.4 I \pm 3.10$ & $6.67 \pm 3.46$ & 0.675 \\
\hline Perioperative NM & $5.68 \pm 2.89$ & $6.23 \pm 2.41$ & $5.98 \pm 2.65$ & 0.280 & $5.90 \pm 3.23$ & $5.97 \pm 2.26$ & $5.93 \pm 2.77$ & 0.917 \\
\hline Perioperative LM & $0.40 \pm 0.48$ & $0.69 \pm 0.51$ & $0.55 \pm 0.52$ & $0.003^{b}$ & $0.49 \pm 0.44$ & $0.60 \pm 0.44$ & $0.55 \pm 0.44$ & 0.322 \\
\hline Perioperative PM & $24.78 \pm 31.20$ & $15.02 \pm 27.39$ & $19.54 \pm 29.48$ & 0.178 & $28.84 \pm 36.72$ & $12.38 \pm 30.57$ & $20.61 \pm 34.53$ & 0.071 \\
\hline Perioperative WBCM & $5.34 \pm 2.8 \mathrm{I}$ & $5.64 \pm 2.43$ & $5.50 \pm 2.6 \mathrm{I}$ & 0.365 & $5.49 \pm 3.03$ & $5.47 \pm 2.12$ & $5.48 \pm 2.60$ & 0.648 \\
\hline ACS & 0 & 1 & 1 & 0.351 & 0 & 0 & 0 & $\mathrm{a}$ \\
\hline PE & 0 & 0 & 0 & a & 0 & 0 & 0 & a \\
\hline $\mathrm{AF}$ & I & 2 & 3 & 0.648 & 0 & 1 & 1 & 0.313 \\
\hline Pneumonia & 0 & 0 & 0 & a & 0 & 0 & 0 & a \\
\hline Air leak & I & 3 & 4 & 0.384 & I & 1 & 2 & 1.000 \\
\hline Chylothorax & I & 0 & 1 & 0.279 & I & 0 & 1 & 0.313 \\
\hline $\begin{array}{l}\text { Postoperative admission to } \\
\text { ICU }\end{array}$ & 2 & 1 & 3 & 0.473 & 2 & 1 & 3 & 0.554 \\
\hline
\end{tabular}

(Continued) 
Table I (Continued).

\begin{tabular}{|c|c|c|c|c|c|c|c|c|}
\hline \multirow[t]{2}{*}{ Variables } & \multicolumn{4}{|l|}{ Pre-match } & \multicolumn{4}{|l|}{ Post-match } \\
\hline & $\begin{array}{l}\text { Sublobar } \\
\text { Resection }\end{array}$ & Lobectomy & SUM & $\begin{array}{l}p \text { - } \\
\text { value }\end{array}$ & $\begin{array}{l}\text { Sublobar } \\
\text { Resection }\end{array}$ & Lobectomy & Sum & $\begin{array}{l}p- \\
\text { value }\end{array}$ \\
\hline $\begin{array}{l}\text { Perioperative ADL scale } \\
\text { scoring margin }\end{array}$ & $15.90 \pm 10.19$ & $17.33 \pm 11.48$ & $16.67 \pm 10.87$ & 0.743 & $16.41 \pm 10.64$ & $17.97 \pm 12.11$ & $17.19 \pm 11.34$ & 0.795 \\
\hline Readmission within 30 days & 2 & I & 3 & 0.473 & I & I & 2 & 1.000 \\
\hline
\end{tabular}

Notes: Duration of operation referred to duration of mono pulmonary ventilation during surgery. ${ }^{\mathrm{a}}$ Means no effective statistical analysis was performed. ${ }^{\mathrm{b}}$ Refers to the number $<0.05$.

Abbreviations: BMI, body mass index; STAS, spread through air spaces; WBC, white blood cell; NLR, neutrophil-lymphocyte ratio; LM, lymphocyte margin; ACS, acute coronary syndrome; PE, pulmonary embolism; AF, atrial fibrillation; ICU, intensive care unit; ADL, activity of daily living.

lymph nodes removed $(p=0.000)$, chest drainage duration $(p=0.031)$, and total chest drainage $(p=0.002)$, while no statistically significant differences were found in number of positive lymph nodes ( $p=1.000$ ), length of postoperative hospital stay $(p=0.056)$, blood test results, complications, postoperative ADL scale scoring margin, and readmission within 30 days.

Postoperative chest drainage is the main concern which both surgeons and patients focused on. Our results showed that total chest drainage had a relation with age (correlation coefficient $0.380, p=0.000$ ), hospitalization costs (correlation coefficient $0.320, p=0.001$ ), number of lymph nodes removed (correlation coefficient $0.505, p=0.000$ ), duration of operation (correlation coefficient 0.489, $p=0.000$ ), postoperative albumin level (correlation coefficient $-0.228, p=0.017$ ) and postoperative NLR (correlation coefficient $0.339, p=0.000$ ). Related results are shown in Figure 1.

Postoperative NLR had a positive relation with total chest drainage (correlation coefficient $0.346, p=0.000$ ), length of postoperative hospital stay (correlation coefficient $0.358, p=0.000$ ), postoperative D-dimer level (correlation coefficient $0.189, p=0.050$ ), and perioperative ADL scale scoring margin (correlation coefficient 0.282, $p=0.003$ ). (Figure 2)

\section{Discussion}

In the past 20 years, thoracic minimally invasive surgery (MIS) has continuously pushed the limit, and enhanced recovery after surgery (ERAS) has gradually gained popularity. To reduce surgical damage and improve the quality of life of patients have gradually become the needs of patients and the goals of surgeons. There has been evidence that patients with VATS gained noninferior long-term survival when compared with patients with thoracotomy for stage I non-small cell lung cancer (NSCLC). ${ }^{6}$ Compared with thoracotomy, MIS was associated with shorter hospital stays, lower pain levels and fewer complications. ${ }^{7-10}$ In recent years, the proportion of VATS in our center has increased, and our patients have recovered well. But there is no convincing evidence about whether different lung resections by means of VATS really have the same minimally invasive effects on patients.

Sublobar resection, including segmentectomy and wedge resection, has gradually emerged, because patients could obtain good lung function retention, ${ }^{11}$ and in patients with early-stage NSCLC, segmentectomy seemed to provide a superior recovery in quality of life compared with lobectomy. ${ }^{12}$ But whether VATS segmentectomy could preserve lung function better than VATS lobectomy in patients with poor lung function remains unclear. ${ }^{13}$ Suzuki et al suggested that no functional advantage for segmentectomy was observed during long-term follow-up, possibly due to compensatory lung growth after lobectomy. ${ }^{14}$ In particular, complex lung segment resection might have limited recovery of residual lung function, resulting in the same loss of lung function as lobectomy. ${ }^{15}$ With regard to cancer prognosis, patients with early-stage lung cancer undergoing segmentectomy showed a similar survival compared with patients undergoing lobectomy. ${ }^{16-}$ ${ }^{19}$ But it is undeniable that there is difference in lymph node dissection between segmentectomy and lobectomy.17,20 Our study supported this opinion. Some studies revealed that, segmentectomy was associated with similar complications compared with lobectomy, except for air leak. ${ }^{21,22}$ Our study suggested that patients showed similar clinical outcomes in postoperative complications, postoperative admission to ICU, ADL scale scores margin, 
A

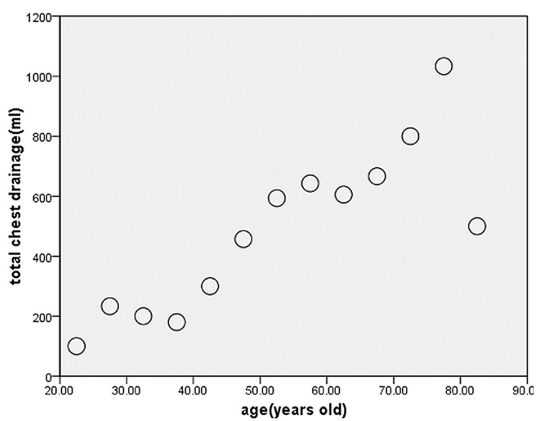

D

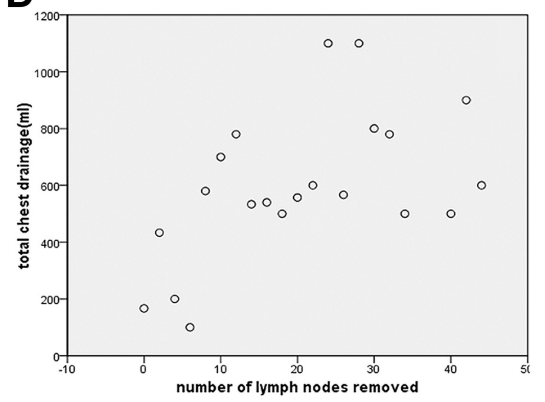

B

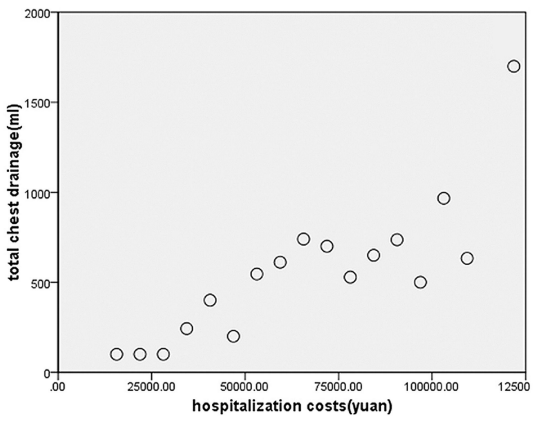

E

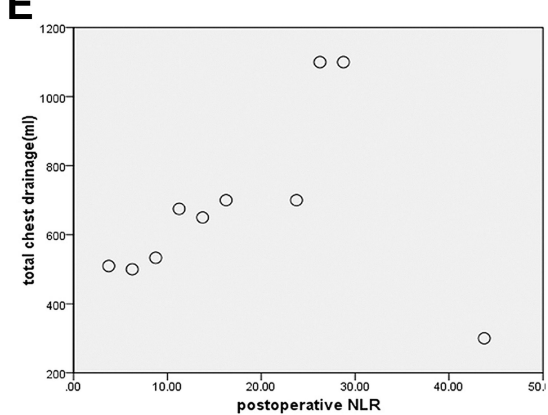

C

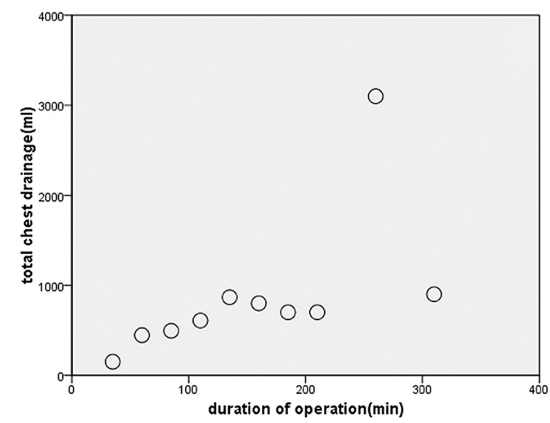

$\mathbf{F}$

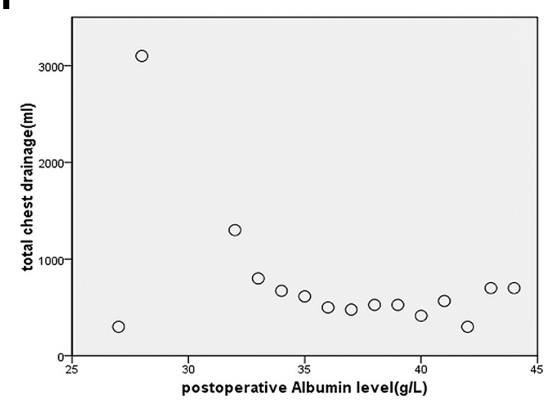

Figure I Rank correlation analysis for chest drainage. Total chest drainage had a positive relation with age (A), hospitalization costs (B), duration of operation (C), number of lymph nodes removed (D) and postoperative NLR (E). Total chest drainage had a negative relation with postoperative albumin level (F).

and readmission within 30 days among different lung resections.

Postoperative inflammatory response can affect patients' immunity. The evaluation of inflammatory response should also be considered in MIS. Many studies have suggested that NLR, as an indicator of inflammatory response, could also affect the tumor microenvironment, thereby affecting tumor prognosis and cytokines released by the tumor microenvironment could also cause neutrophil recruitment. $^{23,24}$ The results of this study showed that perioperative NLR was an important indicator, which was related to total chest drainage, length of postoperative hospital stays, postoperative D-dimer level and perioperative ADL scale scoring margin. No significant difference was found in NLR between different lung resections, although statistical difference in LM was significant. Consequently, we thought different lung resections we performed might share similar inflammatory response and they had the same minimally invasive effect on patients. In addition, based on the results of this study, we hypothesized that NLR could be used to detect the clinical outcome of patients to some extent.

Above all, our results showed that there were differences in chest drainage duration and total chest drainage between different lung resections, but the differences in cost, length of postoperative hospital stay, blood test results, complications in the short-term were not significant. Although there were statistically differences in total chest drainage and chest drainage duration between different lung resections, they were not significantly different from the overall mean, and the inflammatory response shared a similar level between different lung resections. All the lung resections we performed were safe and feasible. Patients underwent different lung resections shared similar shortterm outcomes. Therefore, for the choice of lung resection, we believe that the length of hospital stays and complications cannot be the reasons for sublobar resection.

How to exactly measure the trauma stress and inflammatory response of different lung resections are still what we need to tackle. Whether the so-called MIS can produce minimally invasive effect on patients still needs continuous improvement by surgeons. For cancer treatment, radical tumor resection, as the primary principle, should be considered firstly, while patient's quality of life and

Secondary resectability of pulmonary function (SRPF) should also be weighed. Here, we define SRPF that, for patients with multiple primary lung cancer, the surgeon should try to preserve as much normal lung tissue as possible to ensure that the second primary cancer on the same side would be removable in the future, which also 


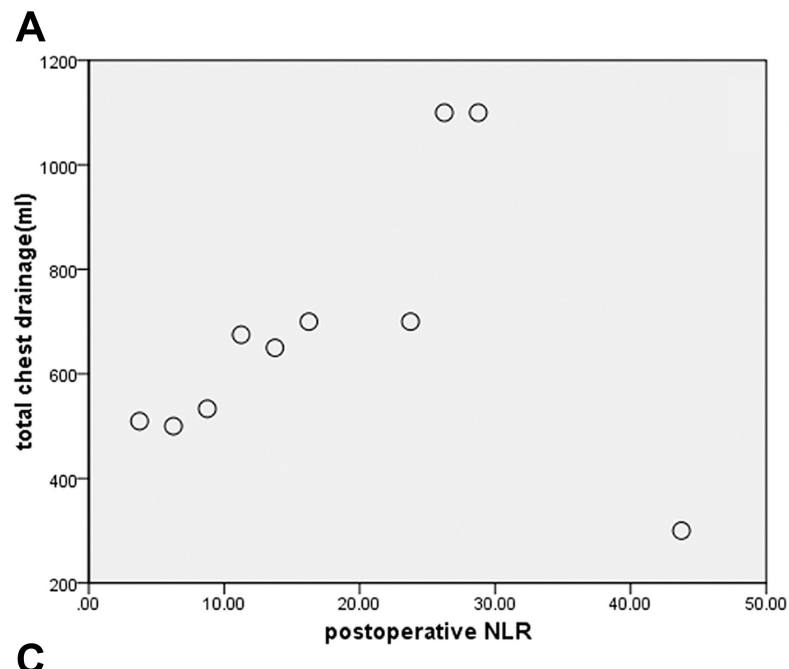

B
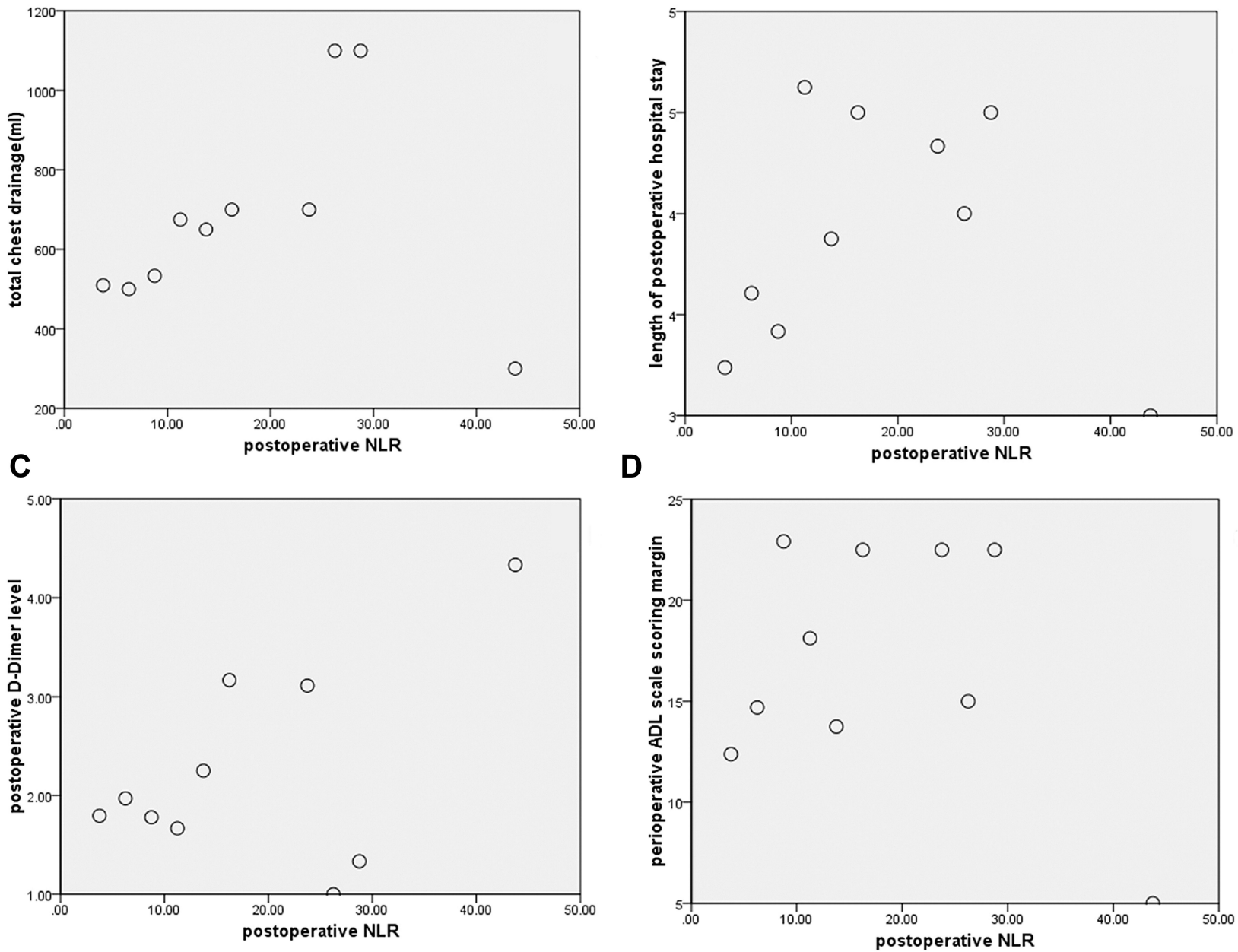

D

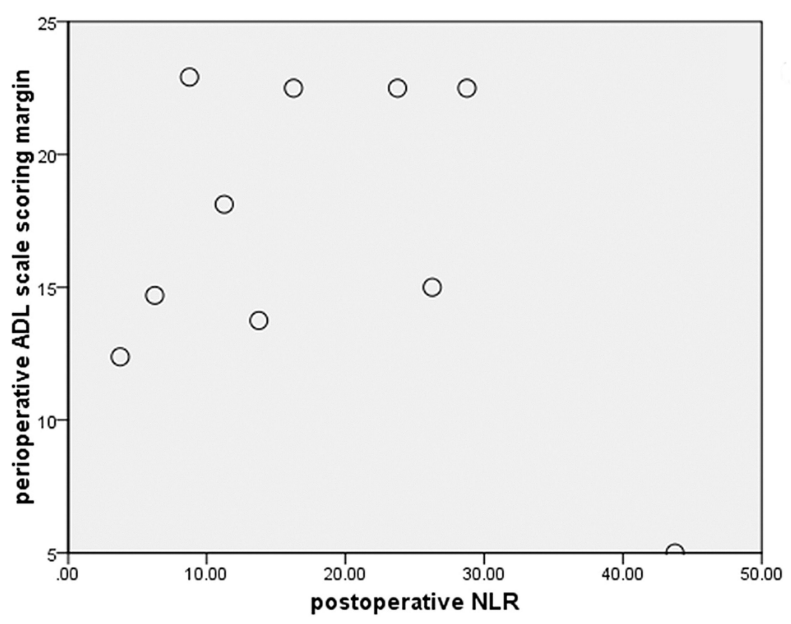

Figure 2 Rank correlation analysis for postoperative NLR. Postoperative NLR had a positive relation with total chest drainage (A), length pf postoperative hospital stay (B), postoperative D-Dimer level (C) and perioperative ADL scale scoring margin (D).

makes patients maintain better quality of life. For the purpose of sublobar resection, SRPF should be the main thinking point. The choice of surgical methods should strictly follow the indications, we must not blindly pursue the so-called minimally invasive effect.

This study has several limitations. Being a single-center retrospective review means it has all the biases based on this study type. Oncological outcomes and long-term effects were unclear. Prognosis for different lung resections may vary and large-scale, multi-center trials are needed.

\section{Conclusions}

Sublobar resection, including wedge resection and segmentectomy, were as safe and feasible as lobectomy in our study. Patients underwent different lung resections shared similar short-term outcomes regarding medical expense, complications, and surgical damage. To some extent, postoperative NLR level could be used to detect the clinical outcomes of patients in critical condition. Indications of lung resection should be standardized to ensure that SRPF is the main purpose of sublobar resection. Long-term oncology comparison between different resections is essential for further instruction.

\section{Ethical Statement}

This study was reviewed and approved by the Institutional Review Board of PUMCH. The authors are accountable for all aspects of the work. Patients were informed of treatment and collection of data. Written informed consent was obtained from all eligible patients.

\section{Acknowledgment}

We would like to give our sincere thanks to nurses in our center for their nursing services. And we want to pay tribute 
to the doctors, nurses, and all workers on the front line for their devotion to the control of novel coronavirus epidemic.

\section{Funding}

There is no funding to report.

\section{Disclosure}

The authors report no conflicts of interest in this work.

\section{References}

1. Bray F, Ferlay J, Soerjomataram I, et al. Global cancer statistics 2018: GLOBOCAN estimates of incidence and mortality worldwide for 36 cancers in 185 countries. CA Cancer J Clin. 2018;68(6):394424. doi:10.3322/caac. 21492

2. Siegel RL, Miller KD, Jemal A. Cancer statistics, 2018. CA Cancer J Clin. 2018;68(1):7-30. doi:10.3322/caac. 21442

3. Siegel RL, Miller KD, Jemal A. Cancer statistics, 2020. CA Cancer J Clin. 2020;70(1):7-30. doi:10.3322/caac.21590

4. Nomori H, Shiraishi A, Cong Y, et al. Differences in postoperative changes in pulmonary functions following segmentectomy compared with lobectomy. Eur J Cardiothorac Surg. 2018;53(3):640-647. doi:10.1093/ejcts/ezx357

5. NCCN Clinical Practice Guidelines in Oncology. Non-Small Cell Lung Cancer, Version 6; 2019.

6. Yang C-FJ, Kumar A, Klapper JA, et al. A national analysis of longterm survival following thoracoscopic versus open lobectomy for stage I non-small-cell lung cancer. Ann Surg. 2019;269(1):163-171. doi:10.1097/SLA.0000000000002342

7. Scott WJ, Allen MS, Darling G, et al. Video-assisted thoracic surgery versus open lobectomy for lung cancer: a secondary analysis of data from the American College of Surgeons Oncology Group Z0030 randomized clinical trial. J Thorac Cardiovasc Surg. 2010;139 (4):976. doi: 10.1016/j.jtcvs.2009.11.059

8. Paul S, Altorki NK, Sheng S, et al. Thoracoscopic lobectomy is associated with lower morbidity than open lobectomy: a propensitymatched analysis from the STS database. $J$ Thorac Cardiovasc Surg. 2010;139(2):366-378. doi:10.1016/j.jtcvs.2009.08.026

9. Falcoz P-E, Puyraveau M, Thomas P-A, et al. Video-assisted thoracoscopic surgery versus open lobectomy for primary non-small-cell lung cancer: a propensity-matched analysis of outcome from the European Society of Thoracic Surgeon database. Eur $J$ Cardiothorac Surg. 2016;49(2):602-609. doi:10.1093/ejcts/ezv154

10. Bendixen M, Jørgensen OD, Kronborg C, et al. Postoperative pain and quality of life after lobectomy via video-assisted thoracoscopic surgery or anterolateral thoracotomy for early stage lung cancer: a randomised controlled trial. Lancet Oncol. 2016;17(6):836-844. doi:10.1016/S1470-2045(16)00173-X

11. Tane S, Nishio W, Nishioka Y, et al. Evaluation of the residual lung function after thoracoscopic segmentectomy compared with lobectomy. Ann Thorac Surg. 2019;108(5):1543-1550. doi:10.1016/j. athoracsur.2019.05.052

Cancer Management and Research

\section{Publish your work in this journal}

Cancer Management and Research is an international, peer-reviewed open access journal focusing on cancer research and the optimal use of preventative and integrated treatment interventions to achieve improved outcomes, enhanced survival and quality of life for the cancer patient.
12. Stamatis G, Leschber G, Schwarz B, et al. Perioperative course and quality of life in a prospective randomized multicenter Phase III trial, comparing standard lobectomy versus anatomical segmentectomy in patients with non-small cell lung cancer up to $2 \mathrm{~cm}$, stage IA (7th edition of TNM staging system). Lung Cancer. 2019;138:19-26. doi:10.1016/j.lungcan.2019.09.021

13. Charloux A, Quoix E. Lung segmentectomy: does it offer a real functional benefit over lobectomy? Eur Respir Rev. 2017;26 (146):170079. doi:10.1183/16000617.0079-2017

14. Suzuki H, Morimoto J, Mizobuchi T, et al. Does segmentectomy really preserve the pulmonary function better than lobectomy for patients with early-stage lung cancer? Surg Today. 2017;47(4):463469. doi:10.1007/s00595-016-1387-4

15. Nomori H, Cong Y, Sugimura H. Systemic and regional pulmonary function after segmentectomy. J Thorac Cardiovasc Surg. 2016;152 (3):747-753. doi:10.1016/j.jtcvs.2016.05.059

16. Zhao Z-R, Situ D-R, Lau RWH, et al. Comparison of segmentectomy and lobectomy in stage IA adenocarcinomas. J Thorac Oncol. 2017;12(5):890-896. doi:10.1016/j.jtho.2017.01.012

17. Kamel MK, Rahouma M, Lee B, et al. Segmentectomy is equivalent to lobectomy in hypermetabolic clinical stage IA lung adenocarcinomas. Ann Thorac Surg. 2019;107(1):217-223. doi:10.1016/j. athoracsur.2018.07.042

18. Razi SS, Nguyen D, Villamizar N. Lobectomy does not confer survival advantage over segmentectomy for non-small cell lung cancer with unsuspected nodal disease. J Thorac Cardiovasc Surg. 2020;159(6):2469-2483 e4. doi:10.1016/j.jtcvs.2019.10.165

19. Song C-Y, Sakai T, Kimura D, et al. Comparison of perioperative and oncological outcomes between video-assisted segmentectomy and lobectomy for patients with clinical stage IA non-small cell lung cancer: a propensity score matching study. J Thorac Dis. 2018;10 (8):4891-4901. doi:10.21037/jtd.2018.07.133

20. Subramanian M, McMurry T, Meyers BF, et al. Long-term results for clinical stage IA lung cancer: comparing lobectomy and sublobar resection. Ann Thorac Surg. 2018;106(2):375-381. doi:10.1016/j. athoracsur.2018.02.049

21. Suzuki K, Saji H, Aokage K, et al. Comparison of pulmonary segmentectomy and lobectomy: safety results of a randomized trial. $J$ Thorac Cardiovasc Surg. 2019;158(3):895-907. doi:10.1016/j. jtcvs.2019.03.090

22. Bedat B, Abdelnour-Berchtold E, Perneger T, et al. Comparison of postoperative complications between segmentectomy and lobectomy by video-assisted thoracic surgery: a multicenter study. $J$ Cardiothorac Surg. 2019;14(1):189. doi:10.1186/s13019-019-1021-9

23. Powell DR, Huttenlocher A. Neutrophils in the tumor microenvironment. Trends Immunol. 2016;37(1):41-52. doi:10.1016/j. it.2015.11.008

24. Kang J, Chang Y, Ahn J, et al. Neutrophil-to-lymphocyte ratio and risk of lung cancer mortality in a low-risk population: a cohort study. Int J Cancer. 2019;145(12):3267-3275. doi:10.1002/ijc.32640
The manuscript management system is completely online and includes a very quick and fair peer-review system, which is all easy to use. Visit http://www.dovepress.com/testimonials.php to read real quotes from published authors. 